\title{
LAT1 (SLC7A5) Overexpression in Negative Her2 Group of Breast Cancer: A Potential Therapy Target
}

\author{
Khaldon Bodoor ${ }^{1 *}$, Rowida Almomani ${ }^{2}$, Mohammad Alqudah ${ }^{3}$, Yazan Haddad ${ }^{4,5}$, \\ Walaa Samouri ${ }^{3}$
}

\begin{abstract}
Objective: HER2 negative carcinomas of the breast pose a challenge for treatment due to redundancies in potential drug targets and poor patient outcomes. Our aim was to investigate the role of L-type amino acid transporter - LAT1 as a potential prognosticator and a drug target. Methods: In this retrospective work, we have studied the expression of LAT1 in 145 breast cancer tissues via immunohistochemistry. Overall survival analysis was used to evaluate patient outcome in various groups of our cohort. Results: Positive LAT1 expression was found in 27 (84.4\%) luminal A subtype, 27 (64.3\%) luminal B/triple positive subtype, 29 (82.9\%) triple negative subtype, and 24 (66.7\%) HER2-only positive subtype ( $\mathrm{p}=0.1$ ). Interestingly, negative correlation was found between LAT1 and HER2; where positive expression of LAT1 was found in $56(83.6 \%)$ cases in negative HER2 group and $51(65.4 \%)$ cases from positive HER2 group $(\mathrm{p}=0.01)$. Unfortunately, we were unable to report significant survival differences when LAT1 expression was studied in the negative HER2 group. Nevertheless, five incidents of mortality (out of 55) were reported in LAT1+/HER2- group compared to none in the LAT1-/HER2- group ( $\mathrm{N}=11)$. Conclusion: Our findings of overexpression of LAT1 in negative HER2 group suggest a role of this protein as prognosticator and drug target in a challenging therapeutic cohort.
\end{abstract}

Keywords: Breast cancer- LAT1-SLC7A5- HER2- ER-PR

Asian Pac J Cancer Prev, 21 (5), 1453-1458

\section{Introduction}

LAT1 (solute carrier family 7 member 5; SLC7A5) is a member of the L-type amino acid transporter family which has 12 transmembrane domains and is covalently associated with solute carrier protein SLC3A2, also known as CD98. LAT1 functions in the bi-directional transport of essential neutral amino acids in and out of cells (Fotiadis et al., 2013). It is known to be overexpressed in multiple types of solid tumors including breast cancer, lung cancer, urogenital cancers, pancreatic cancer, gastrointestinal cancers, head and neck cancers, among others (Hafliger and Charles, 2019). The amino acid transporter LAT1 has recently attracted great attention for its role in breast cancer proliferation and survival (El Ansari et al., 2018a). Breast cancer molecular subtypes, i.e., based on the estrogen receptor (ER), progesterone receptor $(\mathrm{PR})$ and receptor tyrosine-protein kinase erbB-2 (HER2) expression profiles, have helped in unraveling the heterogeneity nature of the disease. In particular, HER2 positive groups (e.g., Luminal A and luminal B subtypes) usually confer good patient outcome compared to the rest. Recent findings suggest potential role of metabolic programming of amino acid transporters including LAT1 in breast cancer heterogeneity (Cha et al., 2018; El Ansari et al., 2018c). Until now, the regulation of LAT1 expression is not well understood, and many transcriptional regulators have been connected with LAT1 overexpression with no common denominator; e.g., c-Myc, hypoxia-inducible factor (HIF2 $\alpha$ ), and regulators of glucose transporters (Scalise et al., 2018).

RNA interference studies have shown that downregulation of LAT1 expression results in growth inhibition in different cancer cell lines. Furthermore, inhibitors of LAT1 are currently being tested in preclinical studies in several types of cancer (Hafliger and Charles, 2019).

Herein, we constructed TMA of 145 samples of breast cancer subtypes to investigate the expression level of LAT1 by immunohistochemistry and its correlation with different clinicopathological parameters.

${ }^{1}$ Department of Applied Biology, Jordan University of Science and Technology, Irbid, Jordan. ${ }^{2}$ Department of Medical Laboratory Sciences, Jordan University of Science and Technology, Irbid, Jordan. ${ }^{3}$ Department of Pathology, Jordan University of Science and Technology, Irbid, Jordan. ${ }^{4}$ Department of Chemistry and Biochemistry, Mendel University in Brno, Zemedelska 1, Brno, Czech Republic. ${ }^{5}$ Central European Institute of Technology, Brno University of Technology, Purkynova, Brno, Czech Republic. *For Correspondence: Khaldon_bodoor@just.edu.jo 


\section{Materials and Methods}

\section{Breast cancer samples}

This study was approved by Faculty of Medicine Research Ethics Committee at Jordan University of Science and Technology (Irbid, Jordan). A retrospective analysis of the pathological records from the department of pathology at King Abdullah University Hospital (Irbid, Jordan) identified 145 female patients diagnosed with breast cancer who underwent radical mastectomy for the tumor or axillary lymph node resection between the years 2007 and 2019.

\section{TMA and Immunohistochemistry}

Tissue microarray (TMA) was constructed from archived paraffin-embedded breast carcinoma tissue blocks using the TMA Master II instrument (3DHISTECH Ltd., Budapest, Hungary). All TMA tissue blocks were sectioned at $4 \mu \mathrm{m}$ thickness and collected on Superfrost plus glass slides for processing by immunohistochemistry. For immunohistochemistry, the BenchMark ULTRA system (Roche Diagnostics, Risch-Rotkreuz, Switzerland) was used to process the TMA slides for immunohistochemistry as previously described (Bodoor et al., 2018). Rabbit polyclonal antibody against LAT1 (SLC7A5) (Thermo Fisher Scientific, Waltham, MA, USA) at 1:100 dilution was used. The slides were scored as described previously (Bodoor et al., 2018) whereas slides with 1-33\% expression were scored as weak and were considered negative in statistical analysis; slides with 34-66\% expression were scored as moderate and were considered positive; and slides with $67-100 \%$ expression were scored as strong and were also considered as positive in statistical analysis. $E R, P R$ and $H E R 2$ expression was taken from the archived records. Tumor volume was calculated differently according to available data for dimensions (For one dimension $\mathrm{x}$ : volume $\left.=4 / 3 * \pi^{*}(\mathrm{x} /)^{2}\right)^{3}$; for two dimensions $\mathrm{x}$ and $\mathrm{y}$ whereas $\mathrm{x}<\mathrm{y}$ : volume $=\mathrm{x}^{2 *} \mathrm{y}$; and for three dimensions $\mathrm{x}, \mathrm{y}$ and $\mathrm{z}$ : volume $=\mathrm{x}^{*} \mathrm{y}^{*} \mathrm{z}$ ).

\section{Statistical Analysis}

Pearson $\chi^{2}$ test of independence was used to compare clinical and pathological characteristics with LAT1 expression. One-sided Fisher's exact test was considered more reliable for $2 \times 2$ crosstabs and thus was reported instead of $\chi^{2}$ test where appropriate. The Kaplan-Meier survival curves were used to represent the overall survival distributions, defined as the period from time of diagnosis to death from any cause or the last contact. The difference in overall survival according to LAT1 expression and clinical and pathological characteristics were analyzed using the log rank test (Mantel, 1966) giving equal weights to individuals at all-time intervals. Data spreadsheet was prepared in Microsoft Excel 2013 (Microsoft, Redmond, WA, USA) and further analysis was done using IBM SPSS Statistics for Windows, Version 21 (IBM Corp, Armonk, NY, USA). p-value $\leq 0.05$ was considered significant.

\section{Results}

Of the 145 patients, 68 women were age 50 years or less while 77 were older than 50 years old. The mean age \pm standard deviation was $51.7 \pm 11.0$ (range 30-82 years). Samples of four subtypes were included in the study; namely, 32 luminal A (ER+/PR+/HER2-), 42 luminal $\mathrm{B}(\mathrm{ER}+/ \mathrm{PR}+/ \mathrm{HER} 2+), 35$ triple negative (ER-/PR-/HER2-), and 36 HER2-only positive (ER-/ PR-/HER2+). LAT1 expression was scored according to

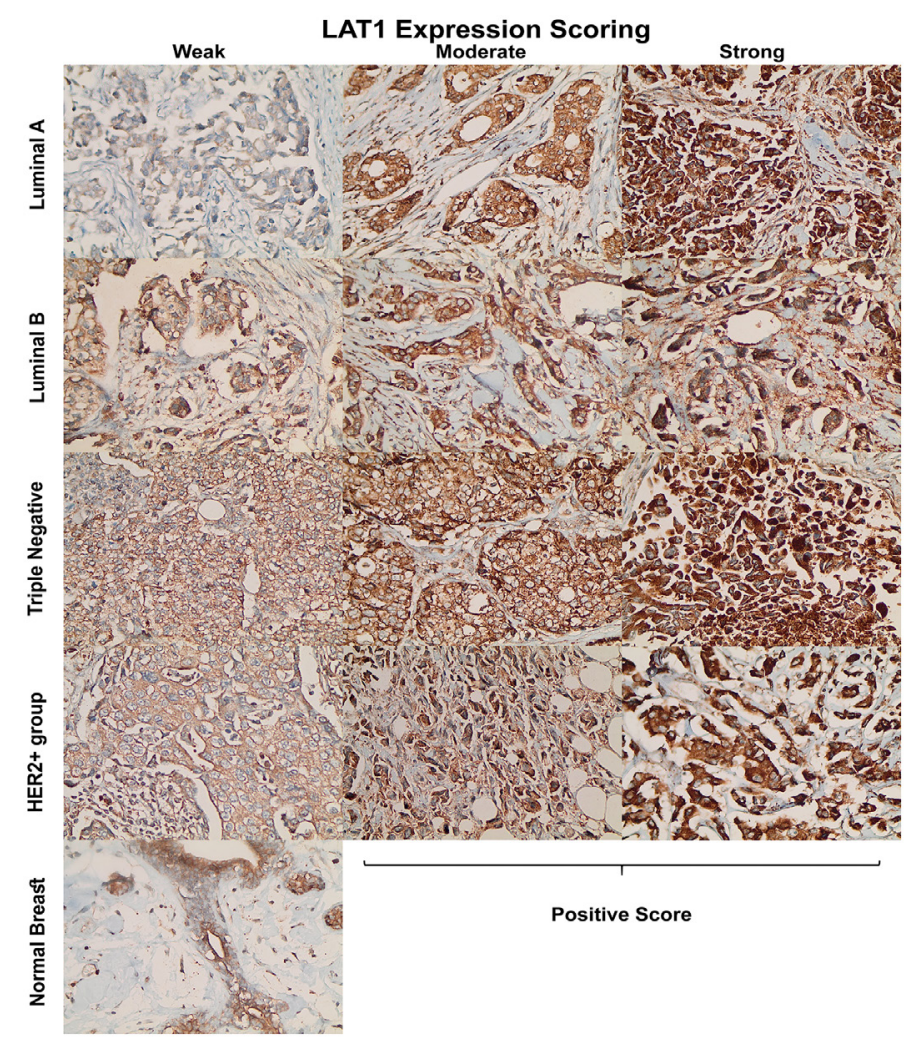

Figure 1. Representative Immunohistochemistry Images for LAT1 Expression and Scoring at 400× Magnification 
DOI: 10.31557/APJCP.2020.21.5.1453 LAT1 (SLC7A5) Overexpression In Negative Her2 Group Of Breast Cancer: A Potential Therapy Target

Table 1. Histopathological, Clinical Characteristics and LAT1 Expression of 145 Breast Cancer Patients. Significant correlation was found between positive LAT1 expression and negative HER2 expression which represents 56 patients of all studied cases.

\begin{tabular}{|c|c|c|c|c|c|}
\hline \multirow[t]{3}{*}{ Characteristic } & \multirow[t]{3}{*}{ Group } & \multicolumn{2}{|c|}{ LAT1 Expression } & \multirow{3}{*}{$\begin{array}{c}\text { Total } \\
\mathrm{N}=145\end{array}$} & \multirow[t]{3}{*}{ p-value } \\
\hline & & Negative & Positive & & \\
\hline & & $\mathrm{N}=38(\%)$ & $\mathrm{N}=107(\%)$ & & \\
\hline \multirow[t]{2}{*}{ Age } & $\leq 50$ years & $20(29.4)$ & $48(70.6)$ & 68 & 0.3 \\
\hline & $>50$ years & $18(23.4)$ & $59(76.6)$ & 77 & \\
\hline \multirow[t]{4}{*}{ Molecular subtypes } & Luminal A & $5(15.6)$ & $27(84.4)$ & 32 & 0.1 \\
\hline & Luminal B (Triple Positive) & $15(35.7)$ & $27(64.3)$ & 42 & \\
\hline & Triple Negative & $6(17.1)$ & $29(82.9)$ & 35 & \\
\hline & HER2-Only Positive & $12(33.3)$ & $24(66.7)$ & 36 & \\
\hline \multirow[t]{2}{*}{$E R / P R$ Expression } & ER-/PR- & $18(25.4)$ & $53(74.6)$ & 71 & 0.5 \\
\hline & $\mathrm{ER}+/ \mathrm{PR}+$ & $20(27.0)$ & $54(73.0)$ & 74 & \\
\hline \multirow[t]{2}{*}{ HER2 Expression } & Negative & $11(16.4)$ & $56(83.6)$ & 67 & $0.01 *$ \\
\hline & Positive & $27(34.6)$ & $51(65.4)$ & 78 & \\
\hline \multirow[t]{5}{*}{ Histological type } & Invasive Ductal Carcinoma & $32(26.2)$ & $90(73.8)$ & 122 & 0.6 \\
\hline & Medullary Carcinoma & $3(42.9)$ & $4(57.1)$ & 7 & \\
\hline & Metaplastic Carcinoma & $1(25.0)$ & $3(75.0)$ & 4 & \\
\hline & Basal or Basal-like Carcinoma & $1(12.5)$ & $7(87.5)$ & 8 & \\
\hline & Micropapillary Carcinoma & $0(0)$ & $3(100)$ & 3 & \\
\hline \multirow[t]{2}{*}{ Ductal Carcinoma in situ (DCIS) } & Absent & $6(20.7)$ & $23(79.3)$ & 29 & 0.3 \\
\hline & Present & $29(29.0)$ & $71(71.0)$ & 100 & \\
\hline \multirow[t]{2}{*}{ Axillary Lymph Nodes } & Negative & $8(22.2)$ & $28(77.8)$ & 36 & 0.3 \\
\hline & Positive & $27(27.8)$ & $70(72.2)$ & 97 & \\
\hline \multirow[t]{2}{*}{ Lymph node Vascular Invasion } & Absent & $8(25.8)$ & $23(74.2)$ & 31 & 0.5 \\
\hline & Present & $23(27.7)$ & $60(72.3)$ & 83 & \\
\hline \multirow[t]{3}{*}{ Tumor volume } & $<10 \mathrm{~cm}^{3}$ & $9(25.7)$ & $26(74.3)$ & 35 & 0.6 \\
\hline & $10-30 \mathrm{~cm}^{3}$ & $12(30.0)$ & $28(70.0)$ & 40 & \\
\hline & $>30 \mathrm{~cm}^{3}$ & $13(21.3)$ & $48(78.7)$ & 61 & \\
\hline \multirow[t]{4}{*}{ Tumor size } & $\mathrm{T} 1$ & $4(50.0)$ & $4(50.0)$ & 8 & 0.08 \\
\hline & $\mathrm{T} 2$ & $19(24.4)$ & $59(75.6)$ & 78 & \\
\hline & $\mathrm{T} 3$ & $8(19.0)$ & $34(81.0)$ & 42 & \\
\hline & $\mathrm{T} 4$ & $7(46.7)$ & $8(53.3)$ & 15 & \\
\hline \multirow[t]{4}{*}{ Lymph node status } & No & $9(24.3)$ & $28(75.7)$ & 37 & 0.2 \\
\hline & N1 & $11(33.3)$ & $22(66.7)$ & 33 & \\
\hline & $\mathrm{N} 2$ & $4(14.3)$ & $24(85.7)$ & 28 & \\
\hline & N3 & $12(35.3)$ & $22(64.7)$ & 34 & \\
\hline \multirow[t]{2}{*}{ Distant metastasis } & M0 & $20(26.0)$ & $57(74.0)$ & 77 & 0.9 \\
\hline & M1 & $14(27.5)$ & $37(72.5)$ & 51 & \\
\hline \multirow[t]{4}{*}{ Stage } & I & $2(50.0)$ & $2(50.0)$ & 4 & 0.7 \\
\hline & II & $11(26.8)$ & $30(73.2)$ & 41 & \\
\hline & III & $6(21.4)$ & $22(78.6)$ & 28 & \\
\hline & IV & $14(27.5)$ & $37(72.5)$ & 51 & \\
\hline \multirow[t]{3}{*}{ Histological grade } & G1 & $2(33.3)$ & $4(66.7)$ & 6 & 0.8 \\
\hline & G2 & $8(22.9)$ & $27(77.1)$ & 35 & \\
\hline & G3 & $28(27.2)$ & $75(72.8)$ & 103 & \\
\hline \multirow[t]{2}{*}{ Family History } & No & $13(26.0)$ & $37(74.0)$ & 50 & 0.3 \\
\hline & Yes & $7(18.9)$ & $30(81.1)$ & 37 & \\
\hline
\end{tabular}

* $p$-value $\leq 0.05$ was considered significant. 
Table 2. Overall Survival Analysis of Differences between the Pathophysiological Groups. Increase in tumor size, lymph node status, metastasis, and lymph node vascular invasion were significantly correlated with poor patient outcome.

\begin{tabular}{|c|c|c|c|c|c|}
\hline Characteristic & Group & $\mathrm{N}$ & Deaths & Mean Survival \pm SE (years) & $p$-value \\
\hline \multirow[t]{2}{*}{ Age } & $\leq 50$ years & 62 & 5 & $10.0 \pm 0.4$ & 0.6 \\
\hline & $>50$ years & 74 & 8 & $12.5 \pm 0.8$ & \\
\hline \multirow[t]{4}{*}{ Molecular subtypes } & Luminal A & 31 & 3 & $9.1 \pm 0.4$ & 1 \\
\hline & Luminal B (Triple Positive) & 41 & 5 & $9.8 \pm 0.5$ & \\
\hline & Triple Negative & 35 & 3 & $9.9 \pm 0.6$ & \\
\hline & HER2-Only Positive & 29 & 2 & $13.7 \pm 0.9$ & \\
\hline \multirow[t]{2}{*}{ ER/PR Expression } & Negative & 64 & 5 & $13.5 \pm 0.6$ & 0.9 \\
\hline & Positive & 72 & 8 & $9.8 \pm 0.4$ & \\
\hline \multirow[t]{2}{*}{ HER2 Expression } & Negative & 66 & 6 & $9.7 \pm 0.5$ & 0.9 \\
\hline & Positive & 70 & 7 & $13.2 \pm 0.7$ & \\
\hline \multirow[t]{5}{*}{ Histological type } & Invasive Ductal Carcinoma & 114 & 12 & $\mathrm{~N} / \mathrm{A}$ & 0.8 \\
\hline & Medullary Carcinoma & 6 & 0 & & \\
\hline & Metaplastic Carcinoma & 4 & 0 & & \\
\hline & Basal or Basal-like Carcinoma & 8 & 1 & & \\
\hline & Micropapillary Carcinoma & 3 & 0 & & \\
\hline \multirow[t]{2}{*}{ Ductal Carcinoma in situ (DCIS) } & Absent & 29 & 5 & $8.1 \pm 0.6$ & 0.09 \\
\hline & Present & 94 & 7 & $11.8 \pm 0.4$ & \\
\hline \multirow[t]{2}{*}{ Axillary Lymph Nodes } & Negative & 36 & 2 & $13.9 \pm 0.8$ & 0.08 \\
\hline & Positive & 89 & 11 & $10.7 \pm 0.7$ & \\
\hline \multirow[t]{2}{*}{ Lymph node Vascular Invasion } & Absent & 28 & 0 & N/A & $0.01 *$ \\
\hline & Present & 78 & 11 & & \\
\hline \multirow[t]{3}{*}{ Tumor volume } & $<10 \mathrm{~cm}^{3}$ & 34 & 1 & $14.0 \pm 0.9$ & 0.2 \\
\hline & $10-30 \mathrm{~cm}^{3}$ & 38 & 5 & $9.6 \pm 0.5$ & \\
\hline & $>30 \mathrm{~cm}^{3}$ & 56 & 6 & $11.5 \pm 0.6$ & \\
\hline \multirow[t]{4}{*}{ Tumor size } & $\mathrm{T} 1$ & 8 & 0 & $\mathrm{~N} / \mathrm{A}$ & 0.2 \\
\hline & $\mathrm{T} 2$ & 72 & 6 & & \\
\hline & $\mathrm{T} 3$ & 40 & 6 & & \\
\hline & $\mathrm{T} 4$ & 14 & 1 & & \\
\hline \multirow[t]{4}{*}{ Lymph node status } & N0 & 37 & 2 & N/A & $0.001 *$ \\
\hline & N1 & 30 & 0 & & \\
\hline & $\mathrm{N} 2$ & 26 & 3 & & \\
\hline & N3 & 31 & 8 & & \\
\hline \multirow[t]{2}{*}{ Metastasis } & None & 77 & 1 & $10.9 \pm 0.1$ & $<0.001^{*}$ \\
\hline & With metastasis & 50 & 12 & $7.3 \pm 0.7$ & \\
\hline \multirow[t]{4}{*}{ Stage } & I & 4 & 0 & N/A & $<0.001 *$ \\
\hline & II & 41 & 0 & & \\
\hline & III & 28 & 1 & & \\
\hline & IV & 50 & 12 & & \\
\hline \multirow[t]{3}{*}{ Histological grade } & G1 & 6 & 0 & N/A & 0.2 \\
\hline & G2 & 34 & 2 & & \\
\hline & G3 & 96 & 11 & & \\
\hline \multirow[t]{2}{*}{ Family History } & No & 49 & 6 & $9.7 \pm 0.5$ & 0.3 \\
\hline & Yes & 37 & 3 & $10.1 \pm 0.5$ & \\
\hline
\end{tabular}

SE, standard error; N/A, not applicable; *, p-value $\leq 0.05$ was considered significant; Statistical analysis was done using Logrank test.

percentage of positive cells (as described in the methods section), and it was categorized into "negative" for both null and weak; and "positive" for both moderate and strong (Figure 1). Positive LAT1 expression was found in 27 (84.4\%) luminal A subtype, 27 (64.3\%) luminal B/triple positive subtype, 29 (82.9\%) triple negative 


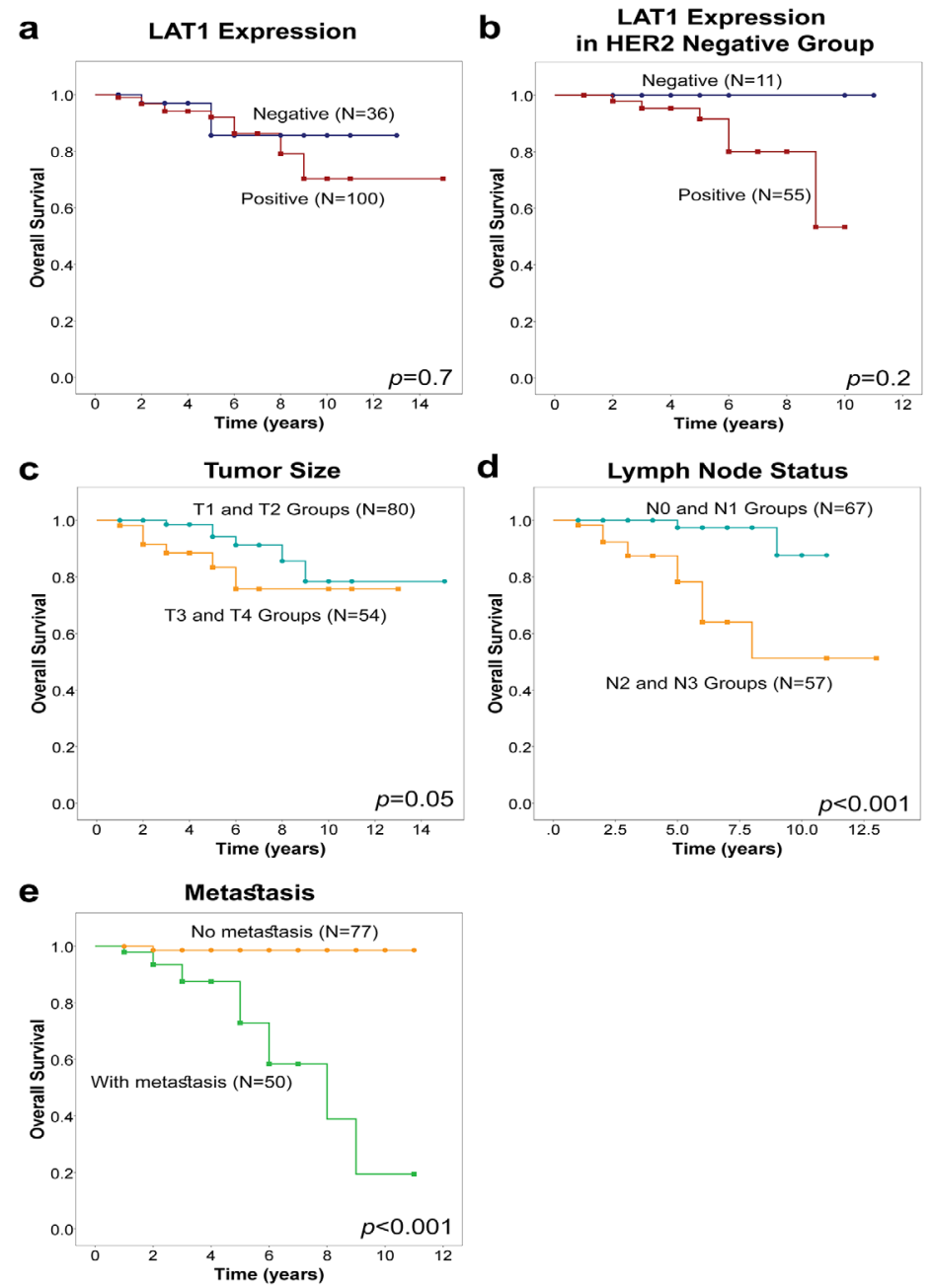

Figure 2. Kaplan-Meier Curves for Overall Survival among Breast Cancer Patients. (a) Overall survival according to LAT1 expression. (b) Overall survival according to LAT1 expression in HER2 negative group. (c) Overall survival according to tumor size. (d) Overall survival according to lymph node status. (e) Overall survival according to metastasis. Overall survival time was defined until event of death from any cause (drop in curve) or last contact (censored cases are shown as circles and squares). $p$-value $\leq 0.05$ was considered significant.

subtype, and $24(66.7 \%)$ HER2-only positive subtypes (Table 1, $\mathrm{p}=0.1$ ). With exception of HER2 expression groups $\left(\chi^{2}\right.$ test $\left.\mathrm{p}=0.01\right)$, no significant correlation was found between $L A T 1$ expression and any of the other clinicopathological characteristics (Table 1). As we have mentioned before, a negative correlation was found between LAT1 and HER2 (Table 1, p=0.01), and we have decided to investigate this further. Positive expression of LAT1 was found in $56(83.6 \%)$ cases from the negative HER2 group and $51(65.4 \%)$ cases from the positive HER2 group. In contrast, survival analysis did not show significant difference between negative and positive LAT1 expression (Figure 2a, $\mathrm{p}=0.7$ ). Furthermore, we investigated overall survival according to $L A T 1$ expression in the negative HER2 group (Figure 2b). Unfortunately, due to the low number of LAT1-/HER2- samples, we were unable to report significant survival differences when LAT1 expression was studied in this cohort. Nevertheless, five incidents of mortality (out of 55) were reported in LAT1+/HER2- group compared to none in the LAT1-/ HER2- group $(\mathrm{N}=11)$.

In contrast, survival analysis (Table 2) showed significant role of some of the histopathological characteristics particularly tumor size (Figure $2 \mathrm{c}, \mathrm{p}=0.05$ ), lymph node status (Figure $2 \mathrm{~d}, \mathrm{p}<0.001$ ) metastasis (Figure $2 \mathrm{e}, \mathrm{p}<0.001$ ) and stage (Table $2, \mathrm{p}=0.004$ ).

Overall, these findings highlight high expression of LAT1 in negative HER2 group, and also show histopathological characteristics (particularly metastasis) as the main determinants of patients' outcome.

\section{Discussion}

Previous genomic and TMA studies of large breast cancer cohorts have shown that LAT1 (SLC7A5) is a prognostic indicator of poor patient outcome in luminal B subtype only (Ring et al., 2006; El Ansari et al., 2018b; El-Ansari et al., 2019; Sevigny et al., 2019). In contrast, Furuya et al., (2012) have shown LAT1 expression is higher in HER2+ and triple negative groups when compared to the luminal A and B subtypes and is associated with poor outcome in triple negative patients. Additionally, Liang et al., (2011) demonstrated that high grade and stage breast cancer tissues (HER2+ and triple negative groups) express higher levels of LAT1. Here we identified relatively high expression of LAT1 
in all subtypes and particularly higher expression in the negative $H E R 2$ groups that are usually more difficult to treat. Although the LAT1 positive group displayed more fatalities, however, that finding was proportional to the number of patients. Here, survival analysis demonstrated clear role of histopathological features related to cancer progression and metastasis but not protein expression (Table 2). Nevertheless, LAT1 high expression in breast cancer provides a new opportunity as a therapeutic target particularly in subtypes that are difficult to treat. Indeed, many strategies targeting LAT1 are under development including potent LAT1 inhibitors (Napolitano et al., 2017; Singh et al., 2018). Alternatively, LAT1 is known to bind cholesterol in the plasma membrane, which can be applied as potential modulation approach to alter its activity (Dickens et al., 2017). LAT1 fits several criteria that are required for identifying cell surface protein targets for use in immunotherapy (Scott et al., 2012) and nanomedicine-based drug delivery (Haddad et al., 2017), such as high expression, accessibility and also identified crystal 3D structure (Yan et al., 2019). Recently, Häfliger and Charles (2019) reviewed current LAT1-targeting therapies particularly the ones in preclinical stage. Our findings support further investigations of this protein for use in treatment of breast cancer, particularly in cohorts that lack any druggable targets.

\section{Acknowledgements}

This work was funded by the Deanship of research of the Jordan University of Science and Technology grant \# 20190103.

\section{Author Contribuiions}

WS and MAperformed TMAand immune-histochemical analysis. YH performed statistical analysis. KB, YH and RA wrote the manuscript. KB was the principal investigator of this work.

\section{Conflict of interest statement}

The authors declare that the research was conducted in the absence of any commercial or financial relationships that could be construed as a potential conflict of interest.

\section{References}

Bodoor K, Abu-Sheikha A, Matalka I, et al (2018). Immunohistochemical analysis of heat shock proteins in triple negative breast cancer: HSP60 expression is a marker of poor prognosis. Eur J Gynaecol Oncol, 39, 926-34.

Cha YJ, Kim ES, Koo JS (2018). Amino acid transporters and glutamine metabolism in breast cancer. Int J Mol Sci, 19, 907.

Dickens D, Chiduza GN, Wright GS, et al (2017). Modulation of LAT1 (SLC7A5) transporter activity and stability by membrane cholesterol. Sci Rep, 7, 43580.

El-Ansari R, Craze ML, Alfarsi L, et al (2019). The combined expression of solute carriers is associated with a poor prognosis in highly proliferative ER+ breast cancer. Breast Cancer Res Treat, 175, 27-38.

El Ansari R, Craze ML, Diez-Rodriguez M, et al (2018a). The multifunctional solute carrier 3A2 (SLC3A2) confers a poor prognosis in the highly proliferative breast cancer subtypes.
Br J Cancer, 118, 1115-22.

El Ansari R, Craze ML, Miligy I, et al (2018b). The amino acid transporter SLC7A5 confers a poor prognosis in the highly proliferative breast cancer subtypes and is a key therapeutic target in luminal B tumours. Breast Cancer Res, 20, 21.

El Ansari R, McIntyre A, Craze ML, et al (2018c). Altered glutamine metabolism in breast cancer; subtype dependencies and alternative adaptations. Histopathology, 72, 183-90.

Fotiadis D, Kanai Y, Palacín M (2013). The SLC3 and SLC7 families of amino acid transporters. Mol Aspects Med, 34, 139-58.

Furuya M, Horiguchi J, Nakajima H, et al (2012). Correlation of L-type amino acid transporter 1 and CD98 expression with triple negative breast cancer prognosis. Cancer Sci, 103, 382-9.

Haddad Y, Heger Z, Adam V (2017). Targeting neuroblastoma cell surface proteins: recommendations for homology modeling of hNET, ALK, and TrkB. Front Mol Neurosci, 10,7 .

Hafliger P, Charles RP (2019). The L-Type amino acid transporter LAT1-an emerging target in cancer. Int J Mol Sci, 20, 2428.

Liang Z, Cho HT, Williams L, et al (2011). Potential biomarker of L-type amino acid transporter 1 in breast cancer progression. Nucl Med Mol Imaging, 45, 93-102.

Mantel N (1966). Evaluation of survival data and two new rank order statistics arising in its consideration. Cancer Chemother Rep, 50, 163-70.

Napolitano L, Scalise M, Koyioni M, et al (2017). Potent inhibitors of human LAT1 (SLC7A5) transporter based on dithiazole and dithiazine compounds for development of anticancer drugs. Biochem Pharmacol, 143, 39-52.

Ring BZ, Seitz RS, Beck R, et al (2006). Novel prognostic immunohistochemical biomarker panel for estrogen receptor-positive breast cancer. J Clin Oncol, 24, 3039-47.

Scalise M, Galluccio M, Console L, et al (2018). The human SLC7A5 (LAT1): The intriguing histidine/large neutral amino acid transporter and its relevance to human health. Front Chem, 6, 243.

Scott AM, Wolchok JD, Old LJ (2012). Antibody therapy of cancer. Nat Rev Cancer, 12, 278-87.

Sevigny CM, Sengupta S, Luo Z, et al (2019). SLCs contribute to endocrine resistance in breast cancer: role of SLC7A5 (LAT1). BioRxiv, 555342.

Singh N, Scalise M, Galluccio M, et al (2018). Discovery of potent inhibitors for the large neutral amino acid transporter 1 (LAT1) by structure-based methods. Int J Mol Sci, 20, 27.

Yan R, Zhao X, Lei J, et al (2019). Structure of the human LAT1-4F2hc heteromeric amino acid transporter complex. Nature, 568, 127-30.

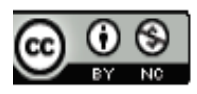

This work is licensed under a Creative Commons AttributionNon Commercial 4.0 International License. 